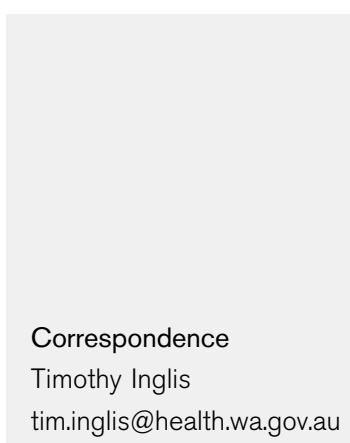

Received 9 May 2007

Accepted 7 September 2007

\section{A hospital-wide study of the impact of introducing a personal data assistant-augmented blood culture round}

\author{
Timothy J. J. Inglis, ${ }^{1}$ Meredith Hodge ${ }^{1}$ and Selva Ketharanathan ${ }^{2}$ \\ ${ }^{1}$ Division of Microbiology and Infectious Diseases, PathWest Laboratory Medicine WA, \\ QEIl Medical Centre, Nedlands, WA 6909, Australia \\ ${ }^{2}$ Department of Medicine, Sir Charles Gairdner Hospital, OEll Medical Centre, Nedlands, WA 6909, \\ Australia
}

\begin{abstract}
Blood culture is the cornerstone of an established aetiological diagnosis of septicaemia. The automated blood culture systems used for this purpose have changed little in the last decade, and the clinical value of results depends on a variety of factors, including pre- and post-analytical variables. Growing scepticism over the value of blood culture results and pressure for the introduction of molecular detection systems have prompted a critical path analysis of pre-, peri- and post-analytical stages in the generation of positive blood culture results. The impact of a positive blood culture was studied in a teaching hospital for 12 months before and 12 months after the introduction of a microbiologist's blood culture round. Active culture reporting via a blood culture ward round was supported by a personal data assistant database of contemporaneous laboratory and clinical data. Hospital occupancy and death register records were subsequently obtained through the State Government data linkage project. There was no evidence that faster laboratory generation of positive blood culture results, faster reporting of results or direct clinical interaction with the patient's primary medical team reduced the risk of death in hospital.

However, there was a threefold increase in the rate of death in hospital following a 1 day delay in collection of blood cultures after hospital admission $(P=0.0010)$. The overall duration of hospital stay for patients with a positive blood culture fell by 2.5 days compared with the previous 12 month period $(P=0.0003)$. The interval between the initial positive culture result and patient discharge fell by 2 days $(P=0.0010)$. This difference was attributed to shorter overall admissions and shorter intervals between positive cultures containing Gram-positive cocci and subsequent patient discharge $(P=0.0018)$. An increased mortality rate from community-acquired bacteraemic infections was associated with delayed culture collection, but not with a prolonged laboratory processing interval. Thus, the speed of conventional blood culture analysis and the form of clinical reporting have little direct effect on the clinical outcome of bacteraemia, but may contribute to a reduction in the length of hospital admission. Introduction of molecular identification tests, such as multiplex PCR methods, at the Gram-stain stage of blood culture is
\end{abstract} unlikely to affect the rate of death in hospital, but may reduce the length of hospital admission.

\section{INTRODUCTION}

The cornerstone of laboratory diagnosis of septicaemia is the detection of bacteria in peripheral venous circulation by blood culture, the principal features of which have remained unchanged for over a decade. During this period, automated processing of blood cultures has become a standard laboratory procedure (Wilson et al., 1994; Mirrett et al., 2003). These systems require an incubation period of at least several hours before the earliest results are available.

Abbreviation: PDA, personal data assistant.
It takes more than $24 \mathrm{~h}$ to complete the entire laboratory process from culture collection to microbial identification and antibiotic susceptibility results (Endimiani et al., 2002). Given this lag between culture collection and definitive result, physicians have become increasingly sceptical of the clinical value of blood cultures (Kelly, 1998; Kennedy et al., 2005; Hall \& Lyman, 2006). Moreover, a positive blood culture does not equate with septicaemia and may not even indicate bacteraemia, if skin bacteria contaminate the contents of the culture bottles during sample collection. There is no completely reliable method to distinguish contaminating bacteria from bona 
fide bacteraemia. Recent improvements in blood culture processing have been made through the molecular detection of staphylococci, enabling rapid recognition of meticillin-resistant Staphylococcus aureus (Fujita et al., 2005). Broader-ranging molecular identification systems, such as gene hybridization and PCR-based probes, have been used to identify bacteria from machine-positive cultures, but these have yet to improve patient outcomes (Lindholm \& Sarkkinen, 2004; Wellinghausen et al., 2004). Other developments in laboratory medicine such as increased reliance on digital reporting or telephone reports from a centralized diagnostic laboratory reduce the direct contact with the patient's attending physician and thus opportunities for result interpretation. A recent study in our Emergency Department questioned the value of indiscriminate early blood culture and led to more selective use of the technique (Mountain et al., 2006). These considerations raise important questions about how a clinical laboratory should organize its blood culture service. In the present study, we aimed to measure the impact of the introduction of a microbiologist's blood culture round.

\section{METHODS}

Blood culture system. All blood cultures during the entire 24 month study period were processed by the Division of Microbiology and Infectious Diseases at the Nedlands campus of PathWest Laboratory Medicine WA. High-fill (10 ml per bottle) blood culture bottles were used throughout, each pair in a single set comprising one aerobic and one anaerobic bottle. After collection in the hospital, these were transferred to the central laboratory reception area for immediate booking in and incubation in an automated blood culture analyser (BACTEC 940; Becton-Dickenson), which is interfaced with the laboratory information system. An aliquot from each machinepositive sample was then centrifuged, and the pellet Gram stained and subcultured onto non-selective laboratory media. Gram-stain and culture results were communicated immediately to the clinical microbiologist on duty for onward reporting to the patient's physician.

No blood culture round or personal data assistant (PDA) database was used during the 12 months prior to the introduction of the PDAaugmented blood culture round. The clinical reporting system used throughout that period was telephone reporting by the Clinical Microbiologist directly to the most immediately available medical practitioner responsible for the patient. Advice was given at this point on the significance of these results, appropriate antibiotic therapy and supplementary diagnostic tests. Clinical microbiology follow-up was performed only on request from the attending physician or when the laboratory generated significant new results such as unexpected antibiotic resistance. The reporting system prior to the introduction of the PDA-augmented blood culture round was therefore reactive telephone reporting and lacked formal clinical audit. A preliminary review (results not shown) indicated a likely discrepancy between clinical microbiology advice given and prescription action taken. Moreover, incomplete record keeping made it difficult to determine accurately the clinical decision-making process consequent to the receipt of an initial blood culture result.

Information systems. Positive blood culture results were studied from a 24 month period in a 600 bed general teaching hospital. A hand-held PDA blood culture database was introduced through a medical microbiology blood culture ward round as a point-of-care decision support tool at the end of the first 12 month period. No other alterations were made to the reporting system, and the majority of clinical microbiologists neither participated in the blood culture round nor used the PDA database. PDA records were continuously updated with laboratory-generated data and records of each interaction with hospital clinical staff. Clinical confidentiality and patient privacy were preserved by avoiding a direct interface between the password-protected hand-held database, the laboratory and the hospital information systems. Database entries were checked against the laboratory's positive blood culture logbook each week. Supplementary antibiotic susceptibility data and definitive identifications were obtained from the electronic laboratory information system within 2 weeks of commencing a database record. The medical microbiology blood culture round took new laboratory data (blood culture Gram-stain results, subsequent identification and susceptibility results, and results from complementary specimens such as mid-stream urine and swab specimens), communicated these to the front-line medical team and obtained clinical management data such as antibiotic usage. A preliminary critical path analysis was conducted into result-reporting pathways on completion of the first 100 consecutive records without interrupting the intervention and reported to clinical teams through grand round lecture presentations, intern induction and medical unit staff meetings. Key messages about optimal presumptive antibiotic therapy were given to clinical teams during the daily blood culture round using a teaching-on-the-run model. On completion of the 24 month data collection period, the blood culture results database was used as a template for death and hospital discharge data from the Government of Western Australian's data linkage system. Data linkage connects records from the contributing datasets such as the Western Australian hospital morbidity system and the death registry with a specified patient. This system reduces the problem of generalization of findings through epidemiological analysis of large datasets (Holman et al., 1999). Positive blood culture results were obtained using the same rules throughout the 24 month study period, which comprised consecutive control and PDA-supported active reporting phases. All positive blood cultures originating from the hospital campus were recorded, including those managed in the Emergency Department and Day Care units.

Statistical analysis. After completion of data entry, the database was converted into spreadsheet format (Excel; Microsoft 2000). Sameday duplicate results were removed. Before stratification, results were restricted to the first positive result per patient. Time intervals were calculated as whole-day differences between admission, sample collection, initial positive result and discharge dates. Numerical data were exported into a biostatistics program to complete the statistical analysis using contingency tables (Fisher's exact), a non-parametric $t$ test (Mann-Whitney U test) and Kaplan-Meier life tables (Prism v2.0; GraphPad).

Definitions. Hospital admission was taken to mean attendance at any component of the hospital campus, including the Emergency Department and day-care units such as the Haematology Care Centre.

A blood culture episode was defined as a positive blood culture from one patient in any $24 \mathrm{~h}$ period. Repeat blood culture results from the same patient on the same date were therefore excluded from further analysis. Outcome analysis was limited to the first blood culture per patient with identical date of birth and hospital reference number.

Early blood culture episodes were positive blood cultures obtained during the first week after hospital admission (days 0-7).

The clinical microbiology reporting pathways identified were the daily blood culture round, the daytime microbiologist and the out-of-hours 
Table 1. General patient outcome and positive blood culture data for two consecutive 12 month periods before (20042005) and after (2005-2006) the introduction of a microbiologist's blood culture round in a tertiary referral hospital

\begin{tabular}{|c|c|c|c|c|}
\hline & \multicolumn{2}{|c|}{ Period } & \multirow[t]{2}{*}{ Total } & \multirow{2}{*}{$\begin{array}{c}P \\
\text { value }\end{array}$} \\
\hline & $\begin{array}{c}2004- \\
2005\end{array}$ & $\begin{array}{c}2005- \\
2006\end{array}$ & & \\
\hline Patients & 484 & 567 & 1051 & NS \\
\hline Positive blood cultures & 599 & 651 & 1250 & NS \\
\hline Median age (years) & 67 & 65 & 66 & NS \\
\hline \multicolumn{5}{|l|}{ Interval between (median days): } \\
\hline Admission and collection & 0 & 0 & 0 & NS \\
\hline Collection and positive result & 0 & 0 & 0 & NS \\
\hline Positive result and discharge & 8 & 6 & 8 & 0.0010 \\
\hline Admission and discharge & 11.5 & 9 & 11 & 0.0003 \\
\hline Death in hospital & 54 & 66 & 119 & NS \\
\hline Discharged to another hospital & 48 & 62 & 110 & NS \\
\hline Discharged home & 333 & 353 & 686 & NS \\
\hline
\end{tabular}

NS, Not significant.

microbiologist. The blood culture round was supplemented by the hand-held clinical database as a clinical decision support tool. The daytime microbiologist was based in the diagnostic laboratory and had access to the laboratory information system but not the PDA database. The out-of-hours microbiologist did not have access to either the handheld database or the laboratory information system.

The information interventions recorded were antibiotic-prescribing advice (start, stop, route of administration, altered duration) and clinical audit (e.g. correction of written record of prior telephone advice, correction of communication breakdown during patient handover at transfer, correction of misreading of laboratory report, curtailment of excessive laboratory investigation).

The outcome death in hospital was defined as death occurring at or before the hospital discharge date. Other types of outcome included discharge to home or discharge to another hospital. The dates given in the public record for these outcomes were used to calculate the time in days from admission to discharge, from admission to blood culture collection, from admission to initial machine culture positive (Gram stain), from collection to positive result, from collection to discharge and from positive result to discharge.

\section{RESULTS}

A total of 1250 positive blood cultures was recorded during the study period: 599 during the first 12 months and 651 during the active reporting phase of the study (Table 1). There was a small rise in the positive result rate from 10.09 to $10.25 \%$, but this was not statistically significant. The number of patients per year with a positive blood culture increased from 484 to 567 . There was no significant change in numbers of patients with positive blood cultures dying in hospital, discharged to another hospital or discharged home. The more common bacterial isolates from blood cultures were broadly similar, with the exception of mixed Gram stains and mixed growth, both of which increased in the active reporting phase, and patients with additional positive culture episodes, which fell during the active reporting phase (Table 2). There was no significant change in the proportion of patients dying in hospital associated with any of the more common causes of bacteraemia.

An outcome analysis of the positive blood cultures indicated that the total number of cultures, and those preceding discharge to home or to another hospital fell rapidly after a peak on the day of admission (Fig. 1). The preponderance of cultures in the first 2 days of hospital admission indicated mainly community-acquired infection. The patients who subsequently died in hospital followed a bimodal distribution, with a significant rise from those whose cultures were collected at admission compared with those cultured the day after admission (day 1) (Fisher's exact test $P=0.0010$ ). Kaplan-Meier analysis of in-hospital survival showed that those with blood cultures collected on the day of admission had a significantly better survival profile than those whose cultures were collected the next day (day 1) (log rank test $P=0.0073$ ) (Fig. 2).

There was no significant difference between the initial and active reporting period in either the proportion of blood culture-positive patients dying in hospital or withinhospital survival times. However, there was a fall in the duration of hospital stay following positive blood culture (Fig. 3; log rank test $P=0.023$ ). This difference was mainly attributable to patients whose cultures contained Grampositive cocci $(P=0.012)$, most of which were coagulasenegative staphylococci. This overall pattern was consistent with differences in the components of blood culture processing for Gram-positive cocci, Gram-negative bacilli and Gram-positive bacilli preliminary results (Table 3).

A proportion of secondary culture results (definitive identification and antibiotic susceptibility results) dealt with on the blood culture ward round followed initial reporting of preliminary results by the daytime or out-of-hours clinical microbiology staff. The possible permutations of two-stage reporting routes produced subgroups too small to assess the modifying impact of antibiotic advice, clinical audit or requests for supplementary information on clinical outcomes. Analysis of the impact of day of culture, collection and duration of culture incubation showed that delayed sample collection was more strongly associated with death in hospital than a prolonged incubation period (Table 4). There was no significant difference in patient age for each of these four groups, nor was there a difference in the proportions of any of the major bacterial species between cultures collected on day 0 and day 1 . The duration of incubation did not correlate with a higher risk of fatality. However, prolonged laboratory incubation did determine to some extent which reporting pathway was followed after the initial Gram-stain result. Prolonged incubation times channelled a disproportionately large number of Grampositive cocci results via the blood culture round. Many of these were subsequently identified as coagulase-negative staphylococci. 
Table 2. Bacteriological results from positive blood cultures for two consecutive 12 month periods before (2004-2005) and after (2005-2006) the introduction of a microbiologist's blood culture round in a tertiary referral hospital

\begin{tabular}{|c|c|c|c|c|c|c|}
\hline \multirow[t]{2}{*}{ Bacteriology } & \multicolumn{2}{|c|}{ Total no. of patients } & \multirow[t]{2}{*}{$P$ value } & \multicolumn{2}{|c|}{ No. of deaths in hospital } & \multirow[t]{2}{*}{$P$ value } \\
\hline & 2004-2005 & 2005-2006 & & 2004-2005 & 2005-2006 & \\
\hline \multicolumn{7}{|l|}{ Initial Gram-stain result: } \\
\hline Gram-positive cocci & 271 & 313 & NS & 32 & 36 & NS \\
\hline Gram-negative bacilli & 151 & 185 & NS & 17 & 20 & NS \\
\hline Gram-positive bacilli & 37 & 40 & NS & 1 & 0 & NS \\
\hline Mixed & 7 & 19 & 0.046 & 0 & 5 & NS \\
\hline Yeasts & 13 & 9 & NS & 4 & 3 & NS \\
\hline \multicolumn{7}{|l|}{ Final culture result: } \\
\hline Coagulase-negative staphylococci & 142 & 140 & NS & 8 & 13 & NS \\
\hline Escherichia coli & 62 & 88 & NS & 7 & 4 & NS \\
\hline Staphylococcus aureus & 57 & 57 & NS & 13 & 10 & NS \\
\hline Klebsiella pneumoniae & 23 & 31 & NS & 1 & 6 & NS \\
\hline Propionibacterium acnes & 22 & 23 & NS & 1 & 0 & NS \\
\hline Streptococcus pneumoniae & 15 & 24 & NS & 2 & 6 & NS \\
\hline Viridans streptococci & 9 & 13 & NS & 0 & 1 & NS \\
\hline Pseudomonas aeruginosa & 11 & 13 & NS & 1 & 3 & NS \\
\hline Enterococcus faecalis & 8 & 15 & NS & 0 & 4 & NS \\
\hline Multiple spp. & 21 & 50 & 0.003 & 4 & 2 & NS \\
\hline Repeat positive results & 89 & 57 & 0.0003 & 9 & 8 & NS \\
\hline Total & 484 & 567 & & 54 & 66 & \\
\hline
\end{tabular}

NS, Not significant.

Analysis of the relationship between the time of the initial report and patient discharge showed that over $10 \%$ of positive results arrived too late to have any influence on decisions during the patient's hospital admission (Fig. 4). For those patients who died, over $12 \%$ of initial positive

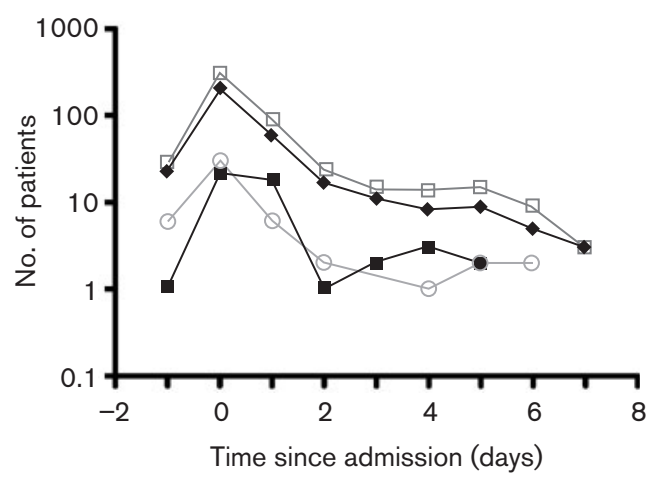

Fig. 1. Frequency distribution diagram of patient outcomes following positive blood cultures in relation to the interval between hospital admission and culture collection, showing a bimodal distribution of positive cultures associated with subsequent deaths in hospital. The difference between death in hospital after blood culture on the admission day and the next day was significant (Fisher's exact test $P=0.0010$ ). $\square$, Total patients; $>$, patients discharged directly into the community; $\bigcirc$, patients discharged to another hospital; $\mathbf{\square}$, patients who died in hospital. blood culture results were not available until after the patient's death in hospital.

\section{DISCUSSION}

Diagnostic microbiology laboratories regard blood culture as one of the most important services they provide. Yet the impact of the current system on patient survival following culture collection is disappointing. In the present study, the earliest laboratory result (Gram stain) followed the

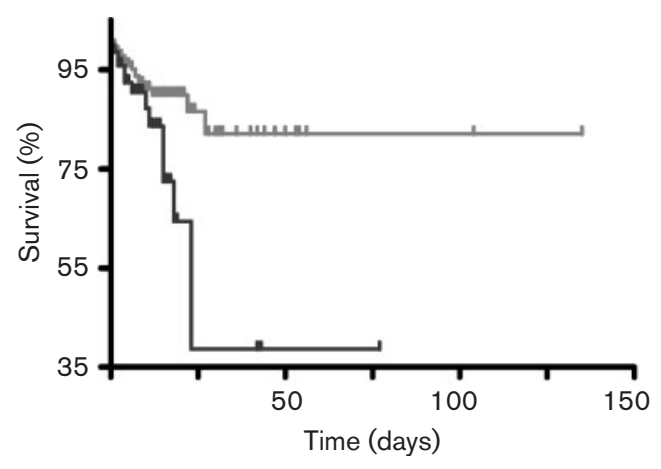

Fig. 2. Kaplan-Meier analysis of in-hospital survival following blood cultures collected on day of hospital admission (day 0) (grey line) compared with cultures collected the following day (day 1) (black line) (log rank test $P=0.0073$ ). 


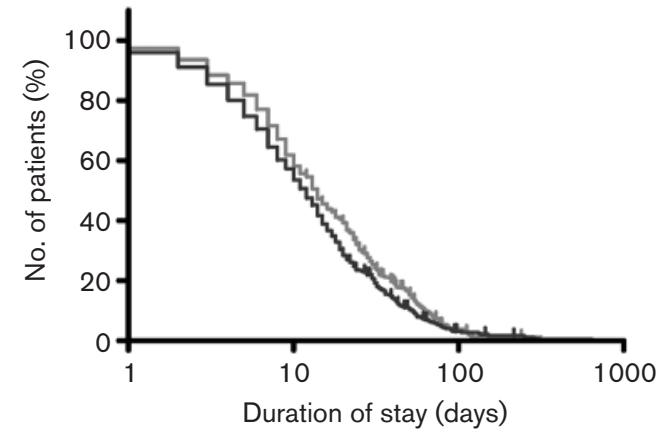

Fig. 3. Kaplan-Meier analysis of duration of hospital stay following initial blood culture result (Gram stain) containing Gram-positive cocci, showing a fall in duration of hospital stay from the control period (February 2004 to February 2005) (grey line) to the active reporting prospective study period (February 2005 to February 2006) (black line). Curves were significantly different by log rank test $(P=0.0127)$.

patient's death in over $12 \%$ of cases and was not even reported until after patient discharge in over $10 \%$ of cases. This reflects the time it takes for bacteria to multiply beyond the detection threshold of an automated analyser. Moreover, Gram-stain results are non-specific. The uncertain clinical significance of Gram-positive cocci in blood cultures is an additional reason why clinicians are ambivalent towards blood culture (Hall \& Lyman, 2006). Neither the type of clinical microbiology reporting of these preliminary results, nor the speed with which they were generated by the laboratory had any measurable impact on whether the patient died in hospital. The higher mortality noted in this study following delayed collection of blood cultures highlights the importance of late clinical recognition of systemic infection as a possible contributor to an adverse outcome. It is unlikely that early blood culture made any direct contribution to the clinical outcome, given a median interval between culture collection and the Gram-stain result of 1 day. However, early collection of blood cultures may serve as a surrogate marker for early clinical interventions, such as presumptive antibiotic therapy. Further work is needed on the early presentation and clinical management of patients with a positive blood culture to understand how patients can benefit from this observation.

The most evident patient benefit was a reduction in the duration of hospitalization. This was much higher than the 0.8 day reduction in the overall hospital population during the same period. The gains were most marked in the Grampositive coccus patient group, and at least partly explained by faster discharge of patients following assessment of the clinical significance of cultures containing coagulasenegative staphylococci. The shorter duration of stay for patients requiring extended periods of intravenous antibiotic treatment for $S$. aureus infection was made possible by a hospital-in-the-home programme. A combination of concerns about meticillin-resistant $S$. aureus, the ambiguity of Gram-stain results (which cannot distinguish S. aureus from coagulase-negative staphylococci) and the time taken to produce antibiotic susceptibility data have led to a cautious presumptive antibiotic approach to an initial report of Gram-positive cocci that often relies on vancomycin, despite the more rapidly bactericidal activity of high-dose isoxazolyl penicillins (Mitchell \& Howden, 2005). Culture-based information at the stage of preliminary (Gram-stain) data did not provide sufficient grounds for refining anti-staphylococcal antibiotic choice. The choice of presumptive anti-staphylococcal antibiotics needs to be reconsidered in view of the high proportion of meticillin-sensitive $S$. aureus infections in this group and our observation that over $12 \%$ of hospital deaths in culture-positive patients occurred before initial blood culture results were available (Khatib et al., 2006). The median time from hospital admission to death was particularly short in both Escherichia coli and Streptococcus pneumoniae fatal bacteraemias, necessitating clinical management decisions long before definitive identification and antibiotic susceptibility results were reported. The frequency with which these two species cause community-acquired bacteraemia highlights the value of standard clinical management pathways for

Table 3. Median intervals between critical steps in the blood culture process, showing differences between the major categories of Gram-stain result, and between the first and second 12 month study period

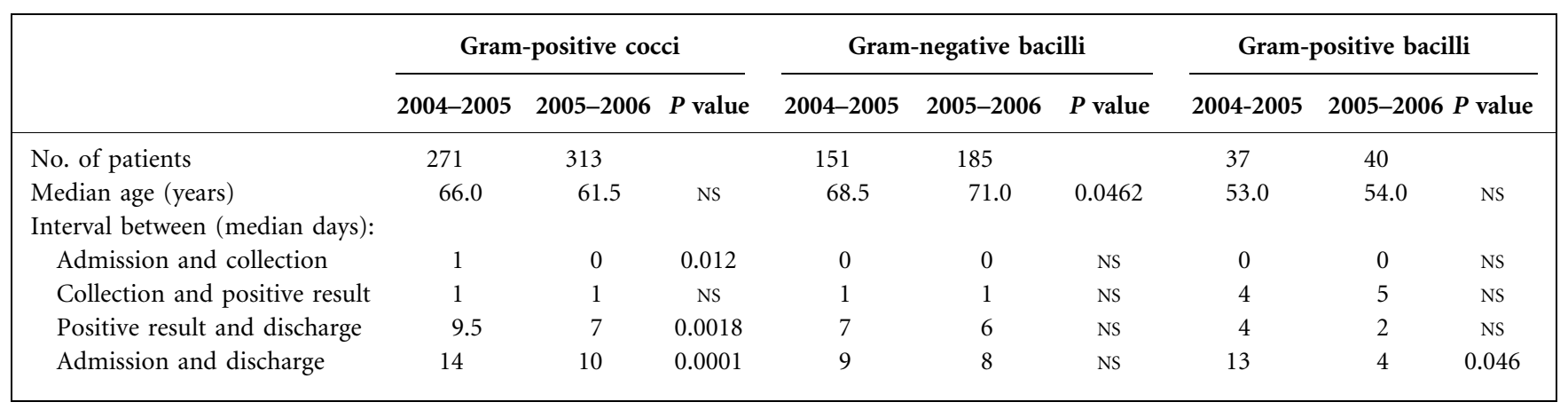

NS, Not significant. 
Table 4. Early blood cultures collected on the day of admission and the next day, reporting pathways and outcomes for the period 2005-2006

\begin{tabular}{|c|c|c|c|c|c|c|c|c|c|}
\hline \multirow[t]{2}{*}{$\begin{array}{l}\text { Timing of } \\
\text { blood culture }\end{array}$} & \multirow{2}{*}{$\begin{array}{l}\text { Interval from } \\
\text { collection to positive } \\
\text { result (days) }\end{array}$} & \multicolumn{3}{|c|}{ Culture result } & \multicolumn{3}{|c|}{$\begin{array}{l}\text { Reporting clinical } \\
\text { microbiologist }\end{array}$} & \multirow{2}{*}{$\begin{array}{c}\text { No. of deaths } \\
\text { in hospital } \\
(\%)\end{array}$} & \multirow{2}{*}{$\begin{array}{l}\text { s Total no. } \\
\text { of patients }\end{array}$} \\
\hline & & $\begin{array}{c}\text { Staphylococcus } \\
\text { aureus }\end{array}$ & $\begin{array}{l}\text { Escherichia } \\
\text { coli }\end{array}$ & $\begin{array}{c}\text { Streptococcus } \\
\text { pneumoniae }\end{array}$ & BCR & DTM & OHM & & \\
\hline \multirow[t]{2}{*}{ On admission } & 0 & 3 & 7 & 3 & 1 & 6 & 22 & $2(7)$ & 30 \\
\hline & 1 & 24 & 42 & 11 & 57 & 53 & 75 & $14(7)$ & 187 \\
\hline \multirow[t]{2}{*}{ Next day } & 0 & 0 & 10 & 3 & 0 & 6 & 13 & $5(26)$ & 19 \\
\hline & 1 & 8 & 6 & 2 & 21 & 8 & 18 & $11(24)$ & 46 \\
\hline
\end{tabular}

BCR, Blood culture round; DTM, daytime microbiologist; OHM, out-of-hours microbiologist.

urosepsis and community-acquired pneumonia. At present, blood culture results are more useful as aggregate epidemiological data than as patient-specific results.

Published studies have examined the effect of antibiotic choice on clinical outcome, and present little data on the process and impact of blood culture reporting practice (Cunney et al., 1997). In our study, the difference in preliminary results reported by different clinical microbiologists reflected a significantly shorter interval between culture collection and the initial detection of Gramnegative bacilli. The peak admission workload of the hospital during office hours resulted in more Gramnegative bacilli reports in daylight hours. The longer time to a positive result for Gram-positive cocci resulted in a peak generation of positive results out of hours. Autoanalyser results generated through the night were read at 7 a.m. the next morning, as in many laboratories without all-hours scientific support. It is unlikely that earlier clinical reporting of a small number of mainly Gram-positive blood cultures significantly affected overall patient outcomes. However, it is clear that this additional

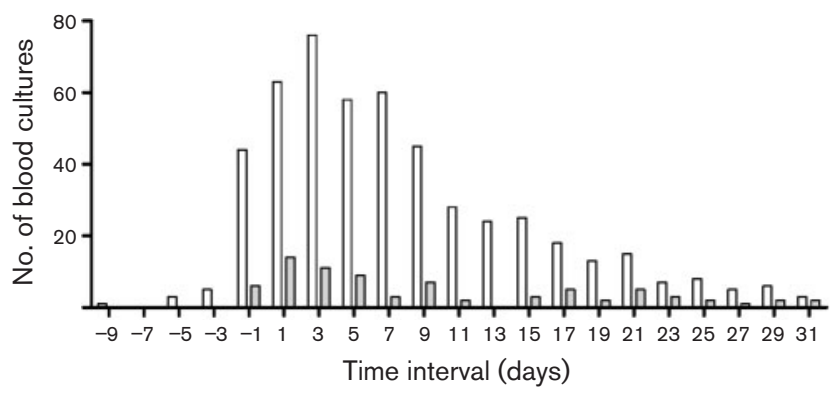

Fig. 4. Distribution of intervals from initial machine-positive blood culture report to patient outcome (discharge or death in hospital). A total of $10 \%$ of all positive results and $12 \%$ of results from patients dying in hospital were reported after these outcomes. Outlier results with an interval of $>31$ days have been omitted. White bars, total positive cultures; grey bars, positive cultures associated with death in hospital. delay weighs against a morning blood culture round achieving a favourable clinical impact. It was predicted some time ago that automation of blood culture analysis would allow laboratories to organize their work around bacterial culture rather than staff availability (Corkill, 1985). It is possible that the clinical impact of positive blood culture could be increased by concentrating face-toface blood culture reporting by the pathologist in the late afternoon and early evening. As the PDA database enabled a more accurate clinical audit, it is not possible to compare the proportion of cases in which antibiotic interventions directed by the clinical microbiologist were effected before and after introduction of the blood culture round. The lack of difference between different reporting pathways during the second 12 month period suggests that the direct contribution may have been relatively small. A further study is required to separate the possible direct effects of PDA database use and face-to-face clinical microbiology consultation at the point of care.

The present study aimed to identify critical points in the blood culture process that might benefit from the range of direct molecular detection tests currently at the planning and validation stage. Our observations highlight the limited clinical benefits of a culture-based system, particularly to the patient. Whilst an understanding of the aetiology of septicaemia can be used as an epidemiological tool in the future management of bacteraemic patients, the delays between recognition of a potential clinical problem and clinically useful results are reason to doubt the immediate utility of blood culture. These results complement earlier observations made in our Emergency Department (Mountain et al., 2006). On the basis of these results, we believe that the proposed introduction of rapid multiplex PCR methods for bacterial detection at the Gram-stain stage of positive blood culture will have little effect on in-hospital mortality (Wellinghausen et al., 2004), but could significantly shorten the duration of hospital stay. Other rapid molecular methods, such as fluorescent in situ hybridization or array-based systems, may have a part to play in the rapid detection of blood-borne bacteria closer to the point of care than conventional blood culture 
(Francois et al., 2006; Peters et al., 2006). It is clear that a reduction in deaths in hospital from community-acquired bacteraemia cannot be obtained within the time frame of a culture-based system. We expect that an improved patient benefit depends on how early the direct molecular detection of bacteraemia can be performed. Studies into the clinical impact of direct molecular detection in early sepsis should be given a high priority and will require a comprehensive clinical outcome analysis.

In conclusion, the speed of conventional blood culture analysis and the form of clinical reporting have little direct effect on the clinical outcome of bacteraemia. However, a microbiologist's blood culture round may reduce the length of hospital admission for some patients with a positive culture. Introduction of molecular bacterial identification tests at the Gram-stain stage of blood culture are unlikely to affect mortality rate, but may reduce the length of hospital admission attributable to bacteraemia. A reduction in mortality rate is only likely if direct molecular detection systems can be used for early laboratory confirmation of bacteraemia shortly after initial presentation.

\section{ACKNOWLEDGEMENTS}

We gratefully acknowledge the contribution of our colleagues at Sir Charles Gairdner Hospital, PathWest Laboratory Medicine WA and the Data Linkage Project of the Government of Western Australia, and particularly Anne Hoskins for clerical assistance with the clinical database, Alison Sewell for data linkage support and Professor Thomas Riley for advice on preparation of this report.

\section{REFERENCES}

Corkill, J. E. (1985). Effects of media, working practice, and automation on the rapid detection of bacteraemia. J Clin Pathol 38, 336-340.

Cunney, R. J., McNamara, E. B., Alansari, N., Loo, B. \& Smyth, E. G. (1997). The impact of blood culture reporting and clinical liaison on the empiric treatment of bacteraemia. J Clin Pathol 50, 1010-1012.

Endimiani, A., Tamborini, A., Luzzaro, F., Lombardi, G. \& Toniolo, A. (2002). Epidemiology of bloodstream infections and time to detection of positive blood cultures: an evaluation of the automated BacT/Alert and BACTEC 9240 systems. New Microbiol 25, 9-16.

Francois, P., Charbonnier, Y., Jacquet, J., Utinger, D., Bento, M., Lew, D., Kresbach, G. M., Ehrat, M., Schlegel, W. \& Schrenzel, J. (2006). Rapid bacterial identification using evanescent-waveguide oligonucleotide microarray classification. J Microbiol Methods 65, 390-403.

Fujita, S., Senda, Y., Iwagami, T. \& Hashimoto, T. (2005). Rapid identification of staphylococcal strains from positive testing blood culture bottles by internal transcribed spacer PCR followed by microchip gel electrophoresis. J Clin Microbiol 43, 1149-1157.

Hall, K. K. \& Lyman, J. A. (2006). Updated review of blood culture contamination. Clin Microbiol Rev 19, 788-802.

Holman, C. D., Bass, A. J., Rouse, I. L. \& Hobbs, M. S. (1999). Population-based linkage of health records in Western Australia: development of a health services linked database. Aust $N Z J$ Public Health 23, 453-459.

Kelly, A. M. (1998). Rationalising the ordering of blood cultures. Aust Health Rev 21, 245-250.

Kennedy, M., Bates, D. W., Wright, S. B., Ruiz, R., Wolfe, R. E. \& Shapiro, N. I. (2005). Do emergency department blood cultures change practice in patients with pneumonia? Ann Emerg Med 46, 393-400.

Khatib, R., Saeed, S., Sharma, M., Riederer, K., Fakih, M. G. \& Johnson, L. B. (2006). Impact of initial antibiotic choice and delayed appropriate treatment on the outcome of Staphylococcus aureus bacteremia. Eur J Clin Microbiol Infect Dis 25, 181-185.

Lindholm, L. \& Sarkkinen, H. (2004). Direct identification of Grampositive cocci from routine blood cultures by using AccuProbe tests. J Clin Microbiol 42, 5609-5613.

Mirrett, S., Reller, L. B., Petti, C. A., Woods, C. W., Vazirani, B., Sivadas, R. \& Weinstein, M. P. (2003). Controlled clinical comparison of BacT/ALERT standard aerobic medium with BACTEC standard aerobic medium for culturing blood. J Clin Microbiol 41, 2391-2394.

Mitchell, D. H. \& Howden, B. P. (2005). Diagnosis and management of Staphylococcus aureus bacteraemia. Intern Med J 35 (Suppl. 2), S17-S24.

Mountain, D., Bailey, P. M., O’Brien, D. \& Jelinek, G. A. (2006). Blood cultures ordered in the adult emergency department are rarely useful. Eur J Emerg Med 13, 76-79.

Peters, R. P., Savelkoul, P. H., Simoons-Smit, A. M., Danner, S. A., Vandenbroucke-Grauls, C. M. \& van Agtmael, M. A. (2006). Faster identification of pathogens in positive blood cultures by fluorescence in situ hybridization in routine practice. J Clin Microbiol 44, 119-123.

Wellinghausen, N., Wirths, B., Franz, A. R., Karolyi, L., Marre, R. \& Reischl, U. (2004). Algorithm for the identification of bacterial pathogens in positive blood cultures by real-time LightCycler polymerase chain reaction (PCR) with sequence-specific probes. Diagn Microbiol Infect Dis 48, 229-241.

Wilson, M. L., Weinstein, M. P. \& Reller, L. B. (1994). Automated blood culture systems. Clin Lab Med 14, 149-169. 